\title{
Orbital Pseudocellulitis: A Retinoblastoma-Associated Masquerade Syndrome
}

\author{
Francesco Martino $^{a} \quad$ Maria Chiara Gelmi $^{a}$ Paolo Galluzzi $^{\text {b }}$ Sonia De Francesco ${ }^{a}$ \\ Clelia Miracco $^{c}$ Doris Hadjistilianou ${ }^{a}$ \\ a Unit of Ophthalmology, Department of Medicine, Surgery and Neuroscience, Azienda Ospedaliera Universitaria \\ Senese, Siena, Italy; ${ }^{b}$ Unit of Neuroimaging and Neurolntervention, Azienda Ospedaliera Universitaria Senese, Siena, \\ Italy; ' Unit of Pathological Anatomy, Department of Medicine, Surgery, and Neurosciences, University of Siena, \\ Siena, Italy
}

\section{Keywords}

Retinoblastoma - Masquerade syndrome - Orbital cellulitis . Pseudocellulitis

\begin{abstract}
Introduction: A masquerade syndrome is an atypical presentation of a neoplastic process that mimics an inflammatory condition. In this paper, we focus on orbital pseudocellulitis. Case Series: Our case series includes 5 retinoblastoma patients with orbital pseudocellulitis at presentation. In 3 patients the disease was bilateral, in 1 trilateral, and in 1 unilateral. The eyes with pseudocellulitis were enucleated, while the fellow eyes were treated conservatively, when affected. Four patients responded well to the therapy and showed remission of the tumor. The patient with trilateral retinoblastoma did not respond to therapy and died of disease. Discussion: Differential diagnosis with infectious orbital cellulitis is extremely important. Patients with orbital cellulitis present with fever, sinusitis, leukocytosis, and raised inflammatory markers, while ophthalmoscopic examination is negative and imaging studies show sinus involvement. On the contrary, patients with retinoblastoma do not show systemic inflammation, while ophthalmoscopic examination reveals leukocoria, buphthalmos, and an intraocular tumor mass as-
\end{abstract}

sociated with retinal detachment. Magnetic resonance imaging shows intralesional calcifications and soft tissue edema without sinus involvement. Histology confirms the diagnosis. Conclusions: Medical history, physical examination, and imaging studies are crucial in the diagnosis of retinoblastoma-associated orbital pseudocellulitis. Retinoblastoma should be excluded in all patients with signs of pre-septal orbital cellulitis through fundoscopy and/or imaging studies

(c) 2020 S. Karger AG, Basel

\section{Introduction}

Retinoblastoma is the most common intraocular tumor in children and its incidence is 1 in 20,000 live births/ year. The typical presentation includes leukocoria and strabismus, but in a minority of cases, retinoblastoma can present as a masquerade syndrome.

A masquerade syndrome is a nonimmune-mediated process that mimics a primary inflammatory condition but is caused by a tumor. Some nonneoplastic processes that can present with inflammatory phenotype have been classified as masquerade syndromes as well, such as infectious uveitis, retinitis pigmentosa, chronic peripheral retinal detachment, or ocular ischemic syndrome. The most karger@karger.com

(c) 2020 S. Karger AG, Basel

www.karger.com/oop

Karger!
Maria Chiara Gelmi

Unit of Ophthalmology, Department of Medicine, Surgery and Neuroscience Azienda Ospedaliera Universitaria Senese

Viale Mario Bracci 16, IL-53100 Siena (Italy)

mariachiara.gelmi@student.unisi.it 
common tumor associated with a masquerade syndrome is intraocular lymphoma, which is typical of the elderly population. Among other conditions, we find melanoma, retinoblastoma, juvenile xanthogranuloma, metastases, and paraneoplastic syndromes [1]. In this paper, we will focus on the retinoblastoma-associated masquerade syndromes, and especially orbital pseudocellulitis.

\section{Retinoblastoma-Associated Masquerade Syndromes}

Retinoblastoma-associated masquerade syndromes can involve the anterior or posterior segment or the extraocular compartment and have a wide spectrum of severity. The inflammation may be caused by intratumoral necrosis attracting lymphocytes or may be simulated by collections of retinoblastoma cells mimicking an iritis, hypopyon, or hyalitis [2]. This type of presentation may lead physicians to treat the cases as uveitis, thereby delaying diagnosis and appropriate treatment. Stafford et al. [3] analyzed 825 histologically confirmed retinoblastoma cases and reported that $6.6 \%$ of them were misdiagnosed as primary ocular inflammation. Balmer et al. [4] analyzed 185 retinoblastoma cases and reported that $14 \%$ had an atypical presentation, with either intraocular or extraocular inflammation.

\section{Pseudo-Anterior Uveitis}

Pseudo-anterior uveitis is caused by the migration into the aqueous humor, ciliary body, iris, lens, and trabecular meshwork of free tumor cells from the peripheral retina. The signs of pseudo-anterior uveitis include chemosis, conjunctival hyperemia, cells in the anterior chamber, and pseudohypopyon [5-8]. Pseudohypopyon is made of tumor cells, moves with changes in head position, and is typically white, while inflammatory hypopyon is usually yellow $[9,10]$. The conjunctival inflammatory signs may be caused by high intraocular pressure (IOP), but they may be absent. The absence of other inflammatory signs is a crucial element in the differential diagnosis between a true hypopyon and a pseudohypopyon, and in this case a retinoblastoma with anterior chamber dissemination should be suspected [10]. Patients with diffuse infiltrating retinoblastoma may show pseudo-uveitis, pseudo-hypopyon, and raised IOP because of the migration of tumor cells from the retinal periphery into the anterior chamber [11]. The differential diagnosis includes several diseases, among which are medulloepithelioma, sarcoidosis, idiopathic uveitis, fungal uveitis, pars planitis, toxocara endophthalmitis, leukemia, lymphoma, and juvenile uveitis associated with rheumatoid arthritis. The definitive diagnosis can be achieved through aqueous humor aspiration, and the treatment consists of enucleation, with or without systemic chemotherapy or radiotherapy [11].

\section{Pseudo-Posterior Uveitis}

A patient with pseudo-posterior uveitis complains of visual loss and floaters and presents with signs of pseudovitritis [5]. While in vitritis the inflammatory debris are irregularly distributed, tumor cells usually form spherical clumps and have a center-sparing distribution [10]. The differential diagnosis is very wide and challenging in this case as well. However, the presence of distinctive spherical clumps in an otherwise quiet eye can be a clue to the correct diagnosis.

\section{Orbital Pseudocellulitis}

Retinoblastoma can present as an extraocular inflammatory condition, as in orbital pseudocellulitis. Orbital inflammation is caused by massive necrosis of intraocular tumor or nontumor tissue and causes eyelid edema, exophthalmos, and frequently neovascular glaucoma. Shields et al. [12] described 5 patients with sporadic retinoblastoma that had been diagnosed and treated as a unilateral infectious orbital cellulitis. After diagnosis of retinoblastoma, the extraocular soft tissue edema evident on computed tomography (CT) was misinterpreted as an extraocular extension of retinoblastoma. Histology showed the tumor tissue was confined to the eyeball and that all cases showed extensive intratumoral necrosis and neovascular glaucoma [13]. Recently, the mechanism behind this inflammatory reaction has been questioned by Ben Meir et al. [14] who examined 4 eyes with intraocular retinoblastoma and orbital inflammation. All 4 eyes showed necrosis of nontumor tissue, that is, anterior segment uvea. The extent of tumor necrosis was variable and completely absent in one case. They concluded that the orbital inflammation may be due to intraocular nontumor tissue necrosis and not to intratumoral necrosis.

The main entity to rule out in the differential diagnosis is infectious orbital cellulitis, and both clinical and radiological findings can help in the process [15]. Patients with infectious orbital cellulitis usually present with fever, leukocytosis, and both clinical and imaging evidence of sinusitis. In pseudo-orbital cellulitis, systemic signs and symptoms are usually absent, there may be only moderate neutrophilic leukocytosis, and there is no evidence of sinusitis, but ophthalmoscopy reveals an intraocular mass with calcifications and other ocular signs, such as a hazy cornea and a high IOP. CT scans show ill-defined soft tissue inflammation and edema, an intraocular mass with calcifications, and no sinus involvement. It is also impor- 
Table 1. Summary of the demographic, clinical, and histological features, and treatment data of the 5 patients in our case series

\begin{tabular}{llllll}
\hline & Case 1 & Case 2 & Case 3 & Case 4 & Case 5 \\
\hline Age at diagnosis & 2 months & 6 months & 10 months & 40 days & 23 months \\
Gender & Female & Female & Female & Female & Male \\
Ethnicity & Caucasian & Caucasian & Caucasian & Caucasian & Caucasian \\
Pseudocellulitis & OD & OD & OS & OD & OS \\
Laterality & Bilateral & Bilateral & Bilateral & Trilateral & Unilateral \\
Family history & Negative & Negative & Positive & Positive & Negative \\
Staging & OD: E (ABC) & OD: E (ABC) & OD: B (ABC) & Trilateral & OD: RTB \\
& OS: B (ABC) & OS: B (ABC) & OS: E (ABC) & OD: E (ABC) & OS: E (ABC) \\
\hline
\end{tabular}

\begin{tabular}{|c|c|c|c|c|c|}
\hline \multicolumn{6}{|l|}{ Histology } \\
\hline Necrosis + microcalcifications & + & + & + & + & + \\
\hline AC, PC, VB involvement & + & + & + & + & + \\
\hline Choroidal/scleral involvement & + & - & + & + & - \\
\hline Focal optic nerve involvement & - & - & + & - & - \\
\hline Extraocular infiltration & - & - & - & - & - \\
\hline \multicolumn{6}{|l|}{ Treatment } \\
\hline Pseudocellulitis & Enucleation & Enucleation & Enucleation & Enucleation & Enucleation \\
\hline Fellow eye & III cycles IR & IV cycles ICE & IV cycles IR & $\begin{array}{l}\text { IV cycles carboplatin- } \\
\text { etoposide + II cycles ICE }\end{array}$ & Uninvolved \\
\hline Outcome & Positive & Positive & Positive & Negative & Positive \\
\hline
\end{tabular}

OD, right eye; OS, left eye; RTB, retinoblastoma.

tant to rule out an extraocular extension of retinoblasto$\mathrm{ma}$, which is rare in developed countries but is still rather common in Saudi Arabia $[16,17]$. In this case, CT scans reveal a distinct extraocular mass without signs of soft tissue inflammation [12].

It is crucial to perform fundus examination in all children with orbital cellulitis, especially pre-septal, in order to rule out a retinoblastoma and, whenever the posterior segment cannot be properly visualized, a CT scan should be performed. The only possible treatment in the case of orbital pseudocellulitis is enucleation, and surgery can be complicated by marked tissue edema. It is possible to reduce tissue edema through preoperative administration of antibiotics and corticosteroids, as we did in all our patients. However, patients who are diagnosed early and treated promptly usually have a good prognosis, especially in the absence of scleral or optic nerve infiltration [15].

\section{Methods}

We conducted a retrospective analysis of our case series from 2000 to 2019. We collected clinical information from the patients' charts stored in the pediatrics department, histological data from the pathological anatomy database, the imaging scans from the neuroradiology program, and the surgical and ophthalmological data from our ophthalmic surgery program.

Children who access our ocular oncology unit are always examined under anesthesia. They are admitted to the pediatrics department and are examined in the ophthalmology operating room. After the examination, the ocular oncologist presents the new cases with a suspected malignancy to the multidisciplinary team, which includes an ocular oncologist, a neuroradiologist, a pediatrician, an interventional radiologist, a pathologist, a geneticist, specialized nurses, and social workers. The specialists in the multidisciplinary team decide the management plan for the patient and organize further examinations and treatment procedures.

Patients with retinoblastoma-associated orbital pseudocellulitis are classified as stage $\mathrm{E}$ retinoblastoma (International Classification Group) and are managed by enucleation as soon as possible. In order to decrease the eyelid edema and make the eyeball easier to access, steroids and systemic antibiotics are started 2 or 3 days before enucleation and are administered for 5 or 6 days. The steroids in use include methylprednisolone $0.5-1.5 \mathrm{mg} / \mathrm{kg} / \mathrm{day}$, and the choice of the antibiotics depends on the age and the health status of the child.

Enucleation is carried out according to the standard guidelines of the no-touch technique, including optic nerve section as posterior as possible. If the contralateral eye is involved, patients are treated with systemic chemotherapy after the enucleation. The chemotherapy regimen is established by the multidisciplinary team according to the tumor characteristics (histopathological risk factors) and the patient's age and clinical features. 
Table 2. Summary of the clinical presentation and ophthalmoscopic findings of the 5 patients in our series of cases

\begin{tabular}{|c|c|c|}
\hline Case & Presentation & Ophthalmoscopy \\
\hline \multirow[t]{2}{*}{1} & $\begin{array}{l}\text { OD: leukocoria, orbital pseudocellulitis } \\
\text { Leukocytosis, negative inflammatory markers }\end{array}$ & OD: buphthalmos, hazy cornea, orbital cellulitis, iris neovascularization \\
\hline & & $\begin{array}{l}\text { OS: ONH and macula free of disease, } 4 \text { tumor foci ( } 1 \text { parapapillary of } 3 \times 3 \mathrm{DD} ; 2 \text { along } \\
\text { infero-temporal branch of } 1 \times 1.5 \mathrm{DD} ; 1 \text { peripheral of } 0.5 \times 0.5 \mathrm{DD})\end{array}$ \\
\hline \multirow[t]{2}{*}{2} & $\begin{array}{l}\text { OD: orbital pseudocellulitis } \\
\text { Leukocytosis, negative inflammatory markers }\end{array}$ & $\begin{array}{l}\text { OD: orbital cellulitis, corneal edema (ophthalmoscopy not possible), secondary } \\
\text { glaucoma }\end{array}$ \\
\hline & & $\begin{array}{l}\text { OS: } 3 \text { tumor foci occupying }>50 \% \text { of the retinal surface (kissing tumor), associated } \\
\text { retinal detachment, and subretinal seeding }\end{array}$ \\
\hline \multirow[t]{2}{*}{3} & & $\begin{array}{l}\text { OD: } \mathrm{ONH} \text { and macula free of disease, } 2 \text { tumor foci in supero-nasal mid-periphery of } \\
6 \times 5 \mathrm{DD} \text { and } 1.5 \times 1.5 \mathrm{DD}\end{array}$ \\
\hline & OS: leukocoria, orbital pseudocellulitis & $\begin{array}{l}\text { OS: shallow AC, rubeosis iridis; OSF: tri-lobed retinal detachment with subretinal } \\
\text { seeding; tumor mass occupies the inferior hemiretina }\end{array}$ \\
\hline \multirow[t]{2}{*}{5} & & OD: uninvolved \\
\hline & OS: leukocoria, orbital pseudocellulitis & $\begin{array}{l}\text { OS: orbital cellulitis, eyelid edema and chemosis, posterior lens dislocation, retinal } \\
\text { detachment with subretinal seeding; tumor mass visible underneath the retinal } \\
\text { detachment }\end{array}$ \\
\hline
\end{tabular}

Inflammatory markers: C-reactive protein, procalcitonin. $\mathrm{ONH}$, optic nerve head; $\mathrm{DD}$, disk diameter.

Fig. 1. Ophthalmoscopy of case 2. Right eye: orbital pseudocellulitis, buphthalmos, and marked corneal edema that does not allow visualization of the fundus.

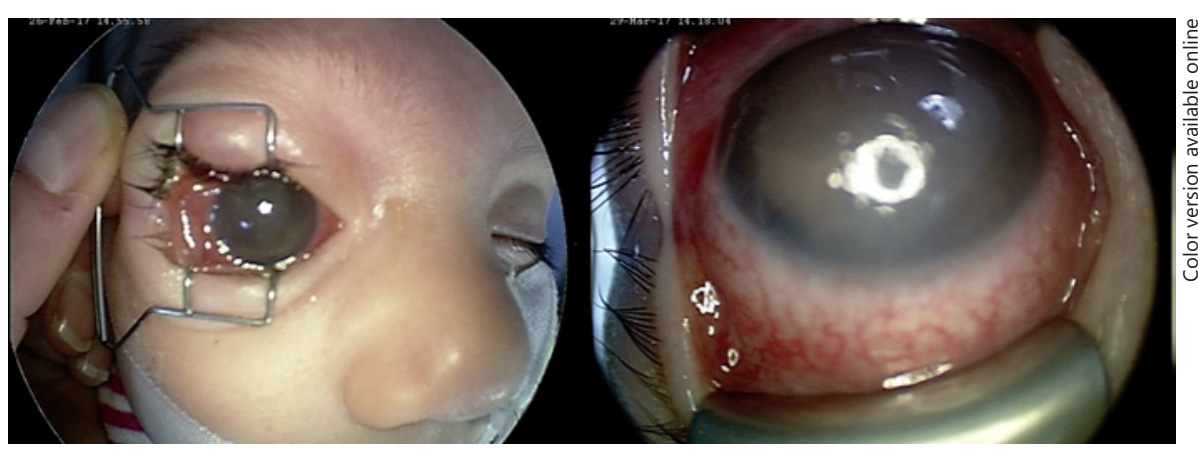

\section{Case Series}

In this paper, we present our case series of 5 patients with retinoblastoma-associated orbital pseudocellulitis who were diagnosed and treated at the University Hospital of Siena. These cases represent about $1 \%$ of the total number of cases examined in our clinic from 1999 to 2019. The parents of all patients gave written informed consent and allowed us to publish their data.

The 5 patients in our series who presented with orbital cellulitis as the first manifestation were between 40 days and 23 months old, 4 out of 5 were female, and all were
Caucasian. Two cases had a retinoblastoma family history. One case had unilateral retinoblastoma, 3 cases had bilateral retinoblastoma, and 1 had metastatic trilateral retinoblastoma. Table 1 contains a summary of the demographic, clinical, and histological features and treatment data of all patients.

\section{Clinical Presentation}

At presentation, all patients had eyelid edema and chemosis, resembling orbital cellulitis, leukocoria, and buphthalmos (shown in Fig. 1). Inflammatory markers were negative and there was moderate leukocytosis. At oph- 


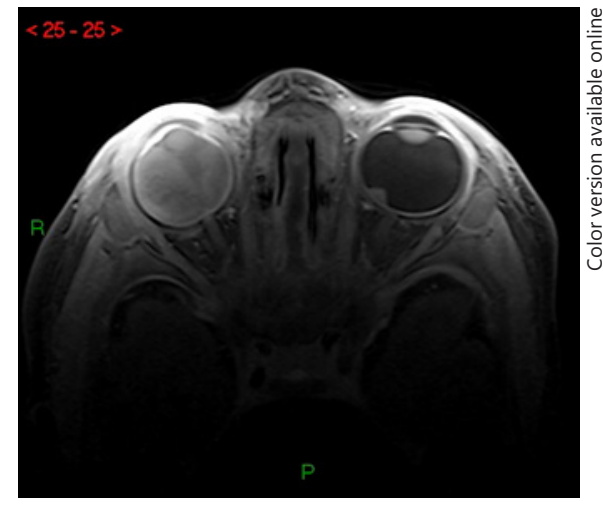

Fig. 2. MRI of case 1, T1 weighted with contrast. Right eye: tumor mass from 2 to 11 o'clock, total retinal detachment, contact with lens and ciliary body, and reduced anterior chamber depth. Intralesional calcifications and necrotic areas. Left eye: 1 tumor focus.

thalmoscopy, the patients had rubeosis iridis, a hazy cornea, and secondary retinal detachment. The contralateral eye showed retinoblastoma foci in the 4 bilateral cases and no involvement in the only unilateral case (case 5). Details of the clinical presentation and ophthalmoscopic features of all patients are outlined in Table 2.

\section{Staging and Diagnosis}

Magnetic resonance imaging (MRI) scans showed the following results. In cases 1 and 2, the eye with orbital cellulitis showed several calcifications and intralesional necrotic areas with low cellularity and moderate enhancement (Fig. 2). Case 3 had a high cellularity index and significant late enhancement washout and slightly altered permeability and perfusion values. Case 4 had trilateral metastatic retinoblastoma, with a lesion in the pineal gland and triventricular hydrocephalon, perimedullary enhancement, and nodular masses in the cauda equina.

The eyes with orbital pseudocellulitis were classified as International Classification Group stage E, the contralateral eyes as International Classification Group stage B, when involved ( 4 out of 5 cases), and case 4 was classified as trilateral.

\section{Histology}

On histology, all 5 cases showed extensive intratumoral necrosis with microcalcifications and infiltration of the anterior chamber. Case 1 had focal scleral infiltration along the course of penetrating vessels, without optic nerve involvement (Fig. 3d-h), and case 3 had focal extrascleral infiltration (Fig. 3a-c) and focal $0.4-\mathrm{mm}$ optic nerve infiltration in the retrolaminar portion, but the re- section margin and other extraocular tissues were free of disease. One element that allows to differentiate a true tumor infiltration from tumor spillage during sectioning is the stromal tissue reaction that is usually present in the context of the tumor infiltration.

\section{Treatment}

All patients underwent enucleation in the eye with orbital pseudocellulitis and conservative treatment in the contralateral eye, when involved. The chemotherapy regimens are reported in Table 1.

\section{Outcome}

The prognosis of our patients was greatly influenced by the early diagnosis performed soon after they reached our center thanks to the integration of a careful clinical examination and imaging studies. Four of our cases had excellent prognosis following enucleation of the eye with pseudocellulitis and conservative treatment of the contralateral eye, when involved. One of our patients had trilateral retinoblastoma with pinealoblastoma and died of disease. Information on response to treatment is reported in Table 1.

\section{Discussion}

Most retinoblastoma cases are diagnosed when the tumor is still intraocular but, as described in a review by Stafford et al. [3], 7\% of cases presented as a masquerade syndrome and were initially diagnosed as primary ocular inflammation. A series of cases by Balmer et al. [4] reported $14 \%$ of cases presenting with atypical signs and symptoms, which included both intraocular and extraocular inflammation. The presence of intraocular or extraocular inflammatory signs, however rare, is responsible for a delay in diagnosis and an increase in mortality $[18,19]$. In more recent series, orbital cellulitis accounted for $4.8 \%$ of retinoblastoma cases in Saudi Arabia and for $5.4 \%$ of cases in Chennai, India [20,21].

Most cases of retinoblastoma presenting with an intraocular inflammatory phenotype are diffuse infiltrating retinoblastomas. Diffuse infiltrating retinoblastoma is a rare variant, is usually unilateral and nonfamilial, and has a later onset (around 6 years of age). The diagnosis of diffuse infiltrating retinoblastoma can be challenging because it lacks a discrete tumor mass and intralesional calcifications, and it is usually achieved through invasive procedures, such as aqueous humor aspiration or fine needle aspiration biopsy. The treatment of this variant of retinoblastoma is usually surgical $[5,13,22,23]$. 


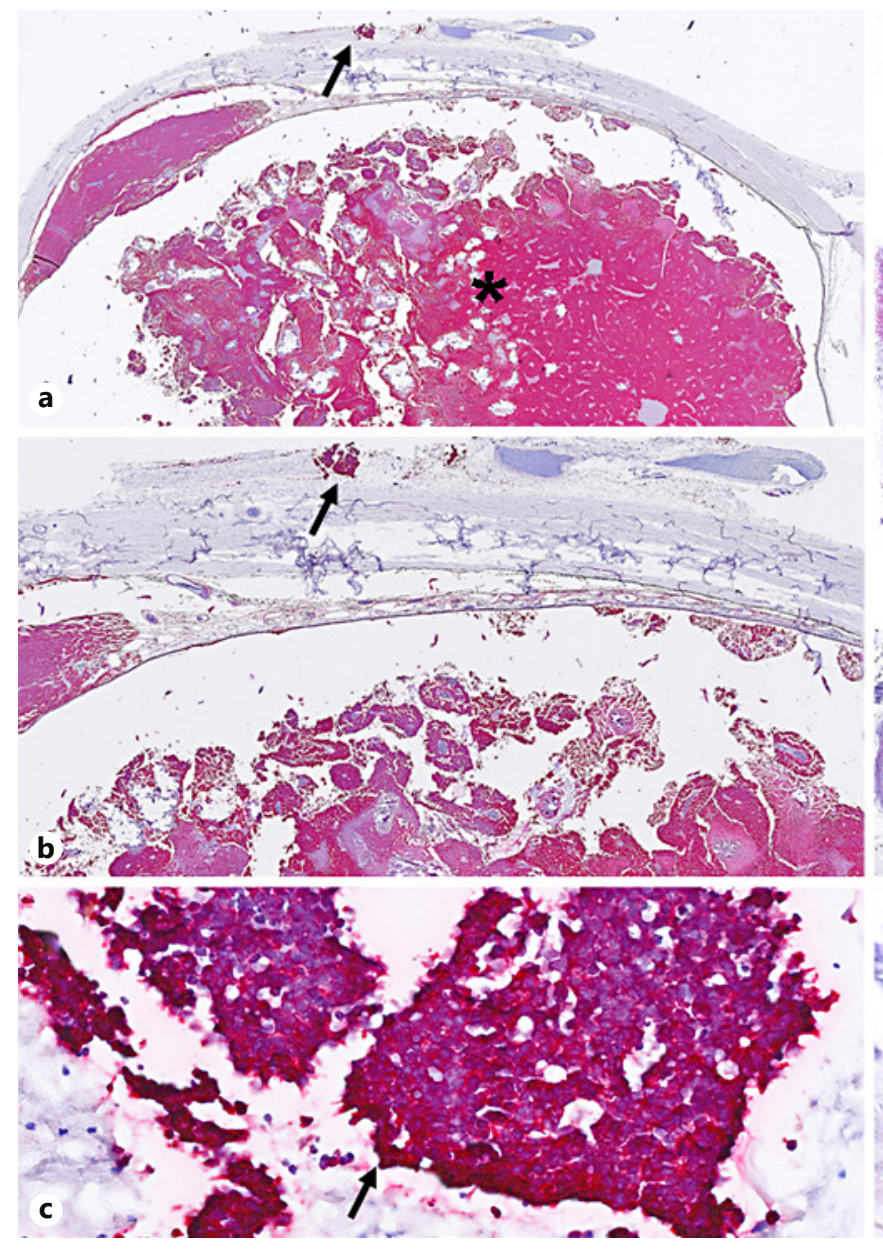

Fig. 3. Histology of cases 1 and 3. a-c Case 3. a The asterisk indicates the intraocular retinoblastoma, positive for the neuronal marker synaptophysin, with vitreal infiltration. The arrow indicates synaptophysin-positive focal extrascleral infiltration (shown at higher magnification in $\mathbf{b}, \mathbf{c}$ ), within a fibrotic stromal reaction. d-h Case 1. synaptophysin-positive intraocular retinoblastoma with extrascleral diffusion (asterisk). The arrowhead indicates the optic nerve, which is also positive for synaptophysin. The upward

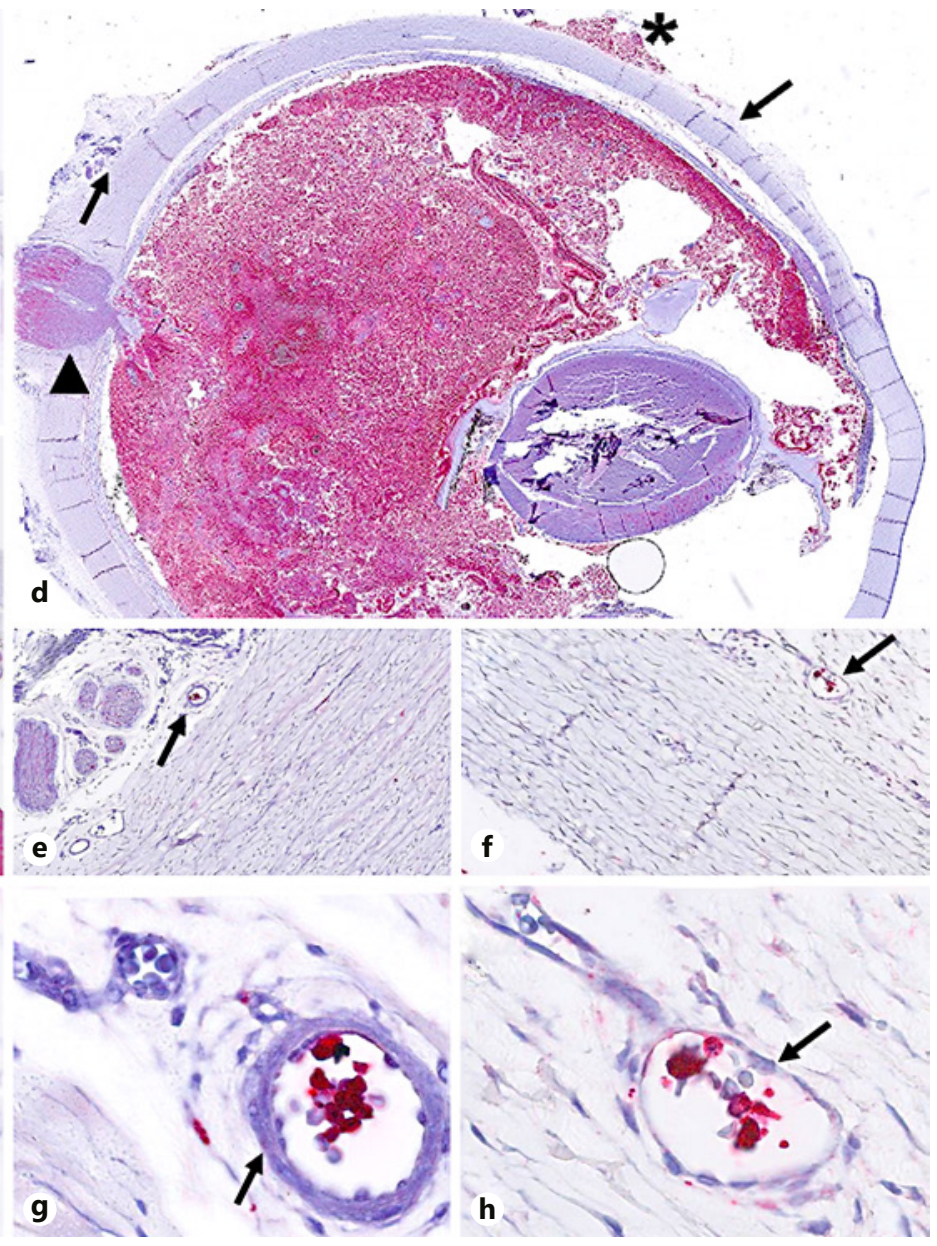

arrow points to an extrascleral vessel (enlarged in e, g). g Synaptophysin-positive retinoblastoma cells inside the vessel lumen. The downward arrow points to an intrascleral vessel (enlarged in $\mathbf{f}, \mathbf{h}$ ), with retinoblastoma cells inside the lumen. Synaptophysin Immunostaining (Ventana Benchmark ULTRA Automatic Immunostainer; Ventana, Roche Diagnostics, Monza, Italy); chromogen new fuchsin, red. Original magnification, $1 \times(\mathbf{a}, \mathbf{d}), 3 \times(\mathbf{b}), 5 \times(\mathbf{e}$, f), $80 \times(\mathbf{c}, \mathbf{g}, \mathbf{h})$.
On the other hand, orbital extension of retinoblastoma is rare in developed countries and, when present, it appears as a discrete extraocular mass on CT or MRI and is not usually associated with inflammatory signs such as eyelid or conjunctival edema. Moreover, orbital extension increases the retinoblastoma-related mortality, even when treated with radiotherapy or chemotherapy [16].

Therefore, it is crucial to differentiate the following clinical pictures: orbital cellulitis, retinoblastoma-associated orbital pseudocellulitis, and orbital extension of retinoblastoma.

Retinoblastoma-Associated Orbital Pseudocellulitis
Both clinical and imaging features can help to differentiate orbital cellulitis from orbital pseudocellulitis. Children with a true orbital cellulitis usually have a history of sinusitis, high temperature, and leukocytosis and have a normal pupil and normal fundus. On the other hand, most patients with intraocular necrotic retinoblastoma do not have fever or marked leukocytosis (our cases showed moderate increase in inflammatory markers with normal WBCs or moderate leukocytosis).

The patients in our series of cases had leukocoria, buphthalmos, or a visible mass at fundoscopy, which was 
evident on MRI and often had intralesional calcifications. The paranasal sinuses were normal or only slightly congested (only one of our cases had marked sinus congestion). Soft tissue edema is evident on MRI. It usually involves the anterior portion of the orbit and is associated with congestion in the optic nerve, but it spares the supero-medial and posterior portions of the orbit, which are closer to the paranasal sinuses and are usually involved in cases of true infectious orbital cellulitis.

In our 4 patients with bilateral retinoblastoma, inflammatory signs were present in one eye only. They had eyelid and anterior orbital inflammation and congestion in the choroid and optic nerve, without tumor extension out of the eyeball except for 1 case with minimal focal extrascleral infiltration, as confirmed by histology.

On histology, case 1 showed scleral infiltration along the course of a penetrating vessel without optic nerve invasion, while case 3 showed a $0.4-\mathrm{mm}$ focal infiltration of the retrolaminar portion of the optic nerve with no infiltration in the resection margin and a minimal focal extrascleral infiltration.

All 5 cases showed extensive intratumoral necrotic areas and anterior chamber infiltration. The cases that had iris neovascularization and angle closure at presentation also had buphthalmos and high IOP.

The mechanism driving retinoblastoma-associated pseudo-orbital cellulitis seems to be a local inflammatory reaction to the extensive intralesional necrosis, caused by rapid tumor growth.

Since most cases of retinoblastoma with orbital pseudocellulitis are very advanced, the appropriate management is enucleation $[21,24]$. In these cases, enucleation is complicated by eyelid edema and should be performed with minimal manipulation and a large lateral canthotomy in order to better remove the eyeball after optic nerve resection. In our patients, eyelid edema was reduced by preoperative administration of antibiotics and corticosteroids. diagnostic delay, and in providing the correct treatment. undergo fundus examination and, whenever the posteri$\mathrm{CT} / \mathrm{MRI}$ in order to exclude the presence of an underlying retinoblastoma.

It is also crucial to correctly interpret imaging studies, especially MRI scans, in order to differentiate signs of extraocular inflammation due to necrosis of an intraocular tumor from retinoblastomas with extraocular extension.

\section{Acknowledgement}

We acknowledge the contributions of ASROO, Scientific Society of Retinoblastoma and Ocular Oncology.

\section{Statement of Ethics}

The study was conducted in accordance with the tenets of the Declaration of Helsinki. The parents of all patients signed a written informed consent and gave the authors permission to publish their data. The study was approved by the Multidisciplinary Oncology Group of the University Hospital of Siena, which includes an ocular oncologist, a pediatrician, a neuroradiologist, a pathologist, an interventional radiologist, a psychologist, a geneticist, and specialized nurses. The Multidisciplinary Oncology Group is an internal organization and it does not assign an ethical approval number to the studies it approves.

\section{Conflict of Interest Statement}

The authors have no conflicts of interest to declare.

\section{Funding Sources}

The authors have no funding sources to declare.

\section{Author Contributions}

D.H. and S.F.: clinical examination and management. P.G.: imaging studies. C.M.: histopathological examination. F.M. and M.C.G.: data collection and analysis; writing of the text.

\section{Conclusions}

In this paper, we have shown that a careful clinical examination and appropriate radiological studies have a crucial role in the differential diagnosis, in reducing the

All children with signs of pre-septal cellulitis should or segment cannot be visualized, ocular ultrasound or

\section{References}

1 Read RW, Zamir E, Rao NA. Neoplastic masquerade syndromes [Review]. Surv Ophthalmol. 2002 Mar-Apr;47(2):81-124.

2 Shields J. Diagnosis and management of intraocular tumors. St Louis: Mosby; 1983. pp. 437-533.

3 Stafford WR, Yanoff M, Parnell BL. Retinoblastomas initially misdiagnosed as primary ocular inflammations. Arch Ophthalmol. 1969 Dec;82(6):771-3.

4 Balmer A, Gailloud C, Munier F, Lendi B, Uffer S. Manifestations inhabituelles du rétinoblastome [Unusual presentation of retinoblastoma]. Klin Monatsbl Augenheilkd. 1994 May;204(5):313-5.
Martino/Gelmi/Galluzzi/De Francesco/ Miracco/Hadjistilianou 
5 Bhatnagar R, Vine AK. Diffuse infiltrating retinoblastoma. Ophthalmology. 1991 Nov; 98(11):1657-61.

6 Schofield P. Diffuse infiltrating retinoblastoma. Br J Ophthalmol. 1960 Jan;44(1):35-41.

7 Shields JA, Shields CL, Eagle RC, Blair CJ. Spontaneous pseudohypopyon secondary to diffuse infiltrating retinoblastoma. Arch Ophthalmol. 1988 Sep;106(9):1301-2.

8 Binder P. Unusual presentations of retinoblastoma. Am J Ophthalmol. 1974;77:674-9.

9 Gritz DC, Hwang DG, Whitcher JP Jr. New horizons in antibacterial therapy. Int Ophthalmol Clin. 1996;36(2):153-70.

10 Nicholson DH. Diffuse infiltrating retinoblastoma: a caveat for the vitreous sur- geon. In: Blodi FC, editor. Contemporary issues in ophthalmology: retinoblastoma. New York: Churchill Livingstone; 1985. pp. 43-61.

11 Jijelava KP, Grossniklaus HE. Diffuse anterior retinoblastoma: A review. Saudi J Ophthalmol. 2013 Jul;27(3):135-9.
12 Shields JA, Shields CL, Suvarnamani C, Schroeder RP, DePotter P. Retinoblastoma manifesting as orbital cellulitis. Am J Ophthalmol. 1991 Oct;112(4):442-9.

13 Andrew JM, Smith DR. Unsuspected retinoblastoma. Am J Ophthalmol. 1965 Sep;60(3): 536-40.

14 Ben Meir A, Bardenstein D, Peiffer R. Retinoblastoma presenting with orbital cellulitis: a mechanical hypothesis. Invest Ophthalmol Vis Sci. 1995;36:492.

15 Foster BS, Mukai S. Intraocular retinoblastoma presenting as ocular and orbital inflammation. Int Ophthalmol Clin. 1996;36(1): 153-60.

16 Ellsworth RM. Orbital retinoblastoma. Trans Am Ophthalmol Soc. 1974;72:79-88.

17 Rootman J, Ellsworth R, Hofbauer J, Kitchen D. Orbital extension of retinoblastoma: a clinicopathological study. Can J Ophthalmol. 1978 Apr;13(2):72-80.

18 Shields JA, Shields C L. Intraocular Tumors. A Text and Atlas, Chap. 18. Philadelphia, W. B. Saunders.
19 Rozansky NM. A necrotic retinoblastoma simulating panophthalmitis. Surv Ophthalmol. 1964 Aug;9:381-3.

20 Mullaney PB, Karcioglu ZA, Huaman AM, alMesfer S. Retinoblastoma associated orbital cellulitis. Br J Ophthalmol. 1998 May;82(5): $517-21$

21 Walinjkar J, Krishnakumar S, Gopal L, Ramesh A, Khetan V. Retinoblastoma presenting with orbital cellulitis. J AAPOS. 2013 Jun; 17(3):282-6.

22 Richards WW. Retinoblastoma simulating uveitis. Am J Ophthalmol. 1968 Mar;65(3): 427-31.

23 Croxatto JO, Fernández Meijide R, Malbran ES. Retinoblastoma masquerading as ocular inflammation. Ophthalmologica. 1983; 186(1):48-53

24 Shields JA, Shields CL, Donoso LA, Lieb WE. Changing concepts in the management of retinoblastoma. Ophthalmic Surg. 1990 Jan; 21(1):72-6. 